\title{
MEMS Thermal Shear-Stress Sensors: Experiments, Theory and Modeling
}

\author{
Qiao Lin, Fukang Jiang, Xuan-Qi Wang, Zhigang Han, Yu-Chong Tai \\ James Lew* and Chih-Ming $\mathbf{H o}^{*}$ \\ Electrical Engineering 136-93, Caltech, Pasadena, CA 91125 \\ * MAE Department, UCLA, Los Angeles, CA 90024
}

\begin{abstract}
We have made many versions of MEMS thermal shear-stress sensors which have received successful applications. However, it has been found that the classical theory for conventional thermal shear-stress sensors is inapplicable to MEMS sensors. This paper then presents a systematic study of this issue and for the first time, an adequate theoretical analysis of MEMS sensors is developed and examined by experiments. The resulting 2D MEMS shearstress sensor theory, which includes heat transfer effects ignored by the classical theory, is verified by experimental data. We also perform 3D heat transfer simulation and the results agree with the testing data and support the proposed new theory.
\end{abstract}

\section{INTRODUCTION}

When a fluid flows over a solid surface, viscous effects generate shear stress at the surface. The measurement of the surface shear stress, which has important applications in fluid dynamics and control [1], can be achieved by thermal sensing methods. This has been demonstrated with several different designs of thermal shear-stress sensors [2-5]. When compared to their conventional counterparts, MEMS sensors are extremely small in size $(\sim 200 \mu \mathrm{m})$ and offer superior spatial resolution and minimal flow interference. Due to improved thermal isolation using a vacuum or air cavity underneath the sensing element (Fig. 1), these sensors are also highly sensitive and consume very little power. While the effort in developing micromachined shear-stress sensors has been successful, a thorough theoretical understanding of the MEMS sensor operation has been lacking. In fact, it has been experimentally observed that our MFMS devices often disagree with the classical hot-wire or hot-film theory, which states that the heat removed by the flow is proportional to the 1/3-power of the shear stress [5]. This suggests that there may be phenomena that the classical theory does not consider, and that a new theory should be developed for the operation of MEMS thermal shear-stress sensors.

This work presents a systematic study including both experimental and theoretical investigations to address this issue. By wind-tunnel testing of various designs of MEMS shear-stress sensors with different membrane thickness, size and material, we first obtain experimental data that confirms the inadequacy of the classical hot-wire/hot-film theory. A more thorough theoretical analysis follows, identifying that this invalidity is due to the lack of a thin thermal boundary layer in the flow. Then, a 2-D MEMS shear-stress sensor theory is developed. We show that by incorporating important heat transfer effects that are ignored by the classical theory, the new model provides a closed-form approximate solution and consistently describes all the MEMS sensors. Moreover, we also present 3D heat transfer simulation that agrees with experiments and supports our new 2-D model. This work demonstrates that many classical assumptions made for conventional thermal devices need to be carefully re-examined for miniature MEMS devices.

\section{MEMS THERMAL SHEAR-STRESS SENSORS}

Fig. 1 illustrates one half of a MEMS thermal shearstress sensor cut along the flow. During operation, a borondoped polysilicon thermistor (used as a hot wire) is heated electrically and maintained at a constant temperature. Since heat transfer rate from the hot wire to the fluid flow is related to the wall shear stress, the power consumption of the hot wire can then be used to determine the shear stress. The hot wire lies on a membrane (typically made of silicon nitride or Parylene), which is in turn suspended over the substrate by a vacuum or air cavity. By improving thermal isolation, the cavity increases sensor sensitivity while lowering power consumption. The detailed fabrication process of these sensors is available in $[2,5]$.

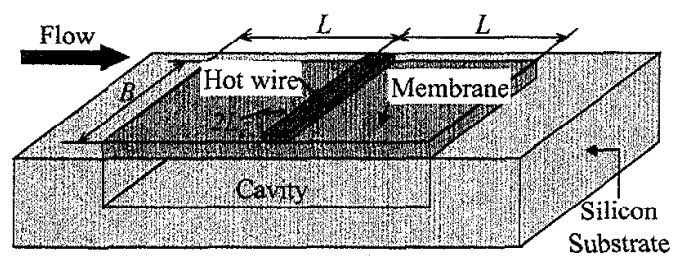

Fig. 1. MEMS shear-stress sensor structure.

\section{EXPERIMENTS}

We have conducted wind-tunnel experiments with four different MEMS shear-stress sensors. Three of the sensors (Fig. 2(a)) have a polysilicon hot wire on a silicon-nitride membrane. These sensors have membrane thickness and hotwire width of $1.5 \mu \mathrm{m}$ and $7 \mu \mathrm{m}$ (the "thin-nitride" sensor), 3 $\mu \mathrm{m}$ and $7 \mu \mathrm{m}$ ("thick-nitride"), and $1.5 \mu \mathrm{m}$ and $3 \mu \mathrm{m}$ ("thinwire"), respectively [2-4]. In the fourth sensor (the "Parylene" sensor), the hot wire (width $=10 \mu \mathrm{m}$ ) is on a Parylene membrane (Fig. 2(b)) [5]. The polysilicon hot 


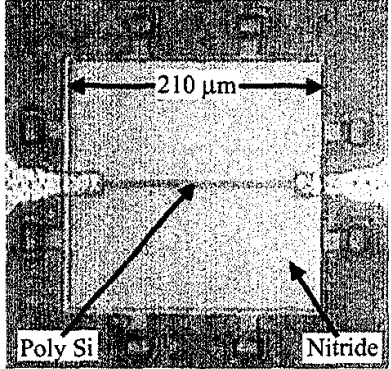

(a)

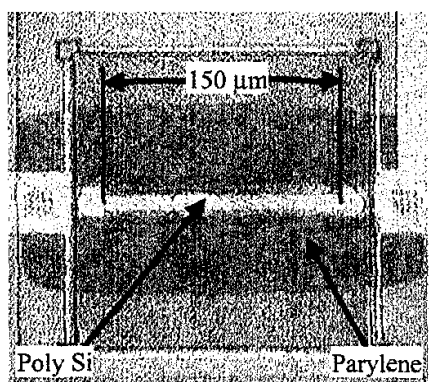

(b)
Fig. 2. MEMS shear stress sensor using (a) nitride and (b) Parylene membranes.

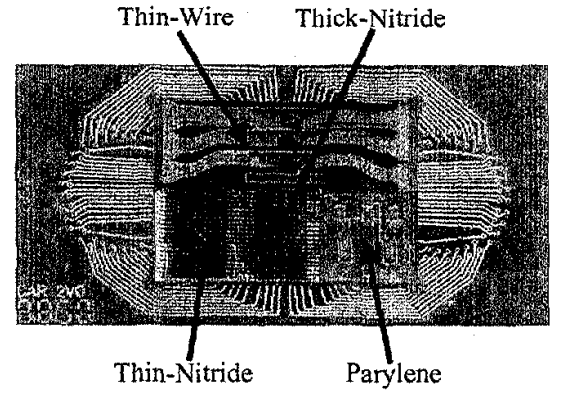

Fig. 3. Four MEMS sensors on the same PCB.

wires in all four sensors have a thickness of $0.5 \mu \mathrm{m}$. These MEMS sensors are carefully placed on a single PC Board (Fig. 3) to ensure uniform testing conditions and consistent testing results. The $\mathrm{PCB}$ is then flush-mounted on the inner wall of a 2-D wind-tunnel [3].

The wind-tunnel testing results are shown in Fig. 4. During sensor operation, the power (denoted $P$ ) needed to maintain the hot wire at a constant temperature depends on the wall shear stress (denoted $\tau$ ). The classical theory states $P-P_{0} \propto \tau^{1 / 3}$, where $P_{0}=\left.P\right|_{\tau=0}$. However, the significantly curved lines in Fig. 4 clearly indicate that this is not true.

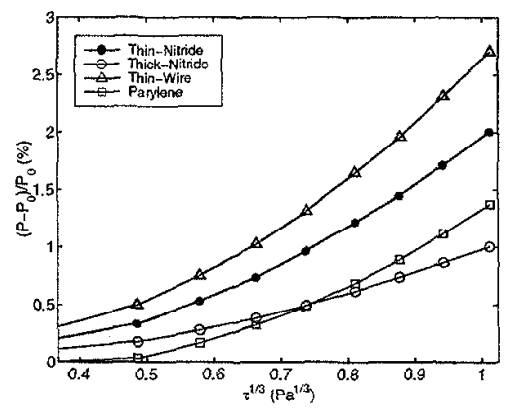

Fig. 4. Measured shear-induced power change vs. $\tau^{1 / 3}$.

\section{INADEQUACY OF THE CLASSICAL THEORY}

To explain the inadequacy of the classical theory, we now carefully examine the classical shear-stress sensor model. This model (Fig. 5) considers a fluid flowing past a plane surface. The temperature at the surface is ambient

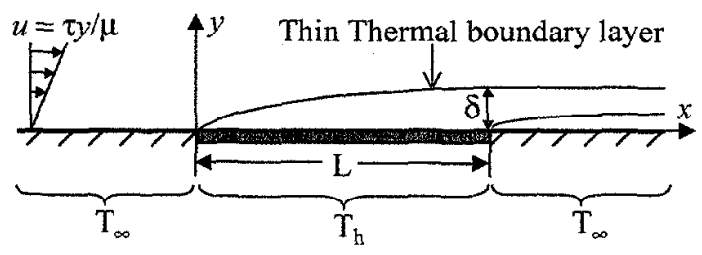

Fig. 5. Classical shear-stress sensor model.

$\left(T_{\infty}\right)$ except over a heated length $L$, where the temperature is $T_{\mathrm{h}}$. Assume $\delta \ll L$, i.e., the thermal boundary layer (the heated region in the fluid) is very thin compared to the heated length. The heat transfer in the flow is then governed by

$$
u \frac{\partial T}{\partial x}=\alpha \frac{\partial^{2} T}{\partial y^{2}},
$$

where $T$ is the fluid temperature and $\alpha$ the fluid's thermal diffusivity. Note that constant material properties are assumed throughout this paper. Under the assumption that the thermal boundary layer is also thin compared with the velocity boundary layer [7], the flow velocity $u$ is linear, i.e.,

$$
u=\frac{\tau y}{\mu}
$$

where $\mu$ is the fluid's dynamic viscosity. Solving this problem yields the classical result that the heat transferred to the fluid over the heated length is proportional to $\tau^{1 / 3}$. To study the validity of the classical theory, we note that with $\bar{\tau}=\tau L^{2} / \mu \alpha$, the thermal boundary layer thickness is given by $\delta / L=0.34 \bar{\tau}^{-1 / 3}$. Thus, for the assumption $\delta \ll L$, and hence the classical theory, to hold, one must have

$$
\bar{\tau}^{1 / 3} \gg 1 \text {. (3) }
$$

For the MEMS sensors used in our experiment, we can readily calculate that $\bar{\tau}^{1 / 3} \leq 2.8$ for the three nitride-based sensors, and $\bar{\tau}^{1 / 3} \leq 1.6$ for the Parylene-based sensor. Thus, for our MEMS sensors, the condition (3) is readily violated and the classical theory is hence invalid.

\section{A NEW THEORY FOR MEMS SENSORS}

Given the failure of the classical theory, a new theory needs to be developed to consistently describe MEMS sensor operation. Clearly, the new theory must allow a thick thermal boundary layer $(\delta \sim L)$. Heat conduction in the membrane is also important, since it can be shown that the abrupt change in the prescribed surface temperature, as assumed in the classical model, is ill-posed in the absence of a thin thermal boundary layer. We use the same notation for fluid properties as used in the classical model.

Our MEMS sensor model is shown in Fig. 6. In this 2D problem, we consider forced convection in the fluid coupled with heat conduction in the membrane (length $=$ $2 L$ ). The membrane's mid-point is maintained at constant temperature $T_{\mathrm{h}}$, representing the hot wire. This essentially ignores the hot wire's streamwise width, which is much 
smaller than $L$. Forced convection in the fluid is then governed by [7]

$$
u \frac{\partial T}{\partial x}=\alpha\left(\frac{\partial^{2} T}{\partial x^{2}}+\frac{\partial^{2} T}{\partial y^{2}}\right)
$$

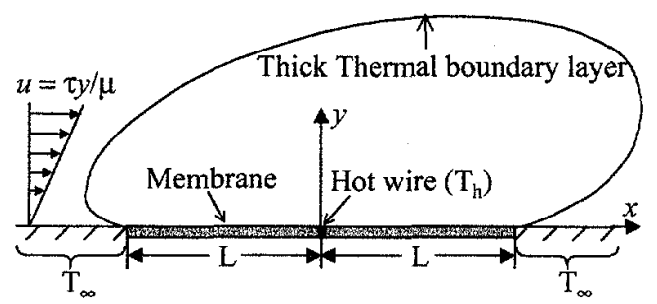

Fig. 6. MEMS shear-stress sensor model.

Comparing Equations (1) and (4), we see that the classical theory ignores the contribution from streamwise heat conduction, which is however very important for MEMS sensors due to the lack of a thin thermal boundary layer. On the other hand, the flow velocity profile (2) is still valid due to the small sensor size.

Heat conduction in the membrane is governed by

$$
k_{m} t \frac{\partial^{2} T_{m}}{\partial x^{2}}+\left.k \frac{\partial T}{\partial y}\right|_{y=0}=0
$$

where $T_{\mathrm{m}}$ is the membrane temperature averaged over the thickness $t(t<L L)$, and $k_{\mathrm{m}}$ is the membrane's thermal conductivity. The coupled heat transfer problem is closed by the following boundary conditions: $T=T_{\mathrm{h}}$ for $x=0$ and $y$ $=0 ; T=T_{\infty}$ for $|x|>L$ and $y=0$ as well as for $x \rightarrow \pm \infty$ or $y$ $\rightarrow+\infty$; and $T_{\mathrm{m}}=T$ as $|x| \leq L$ and $y=0$.

While the solution to this model is generally not in closed form, its functional form can be identified. Define three dimensionless parameters by

$$
\bar{\tau}=\frac{\tau L^{2}}{\mu \alpha}, \quad \bar{P}=\frac{P}{2 k\left(T_{0}-T_{\infty}\right) B}, \quad \text { and } \quad \lambda=\frac{k_{m} t}{k L},
$$

where $B$ is the sensor's transverse length (Fig. 1). It can be shown that the solution to the MEMS sensor model is given by the dimensionless power $\bar{P}$ as a function of the thermal conductivity ratio $\lambda$ and the dimensionless shear stress $\bar{\tau}$. That is, the solution has the functional form

$$
\bar{P}=\bar{P}(\lambda, \bar{\tau})=\bar{P}_{0}(\lambda)+\Delta \bar{P}(\lambda, \bar{\tau})
$$

where we have decomposed the dimensionless power into two components: the power in still fluid $\bar{P}_{0}=\bar{P}(\lambda, 0)$, and

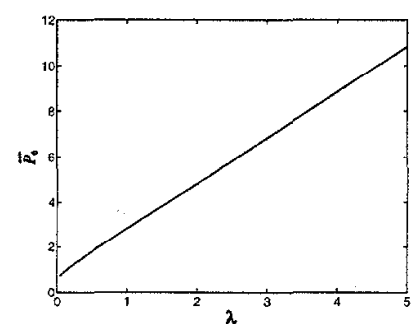

(a)

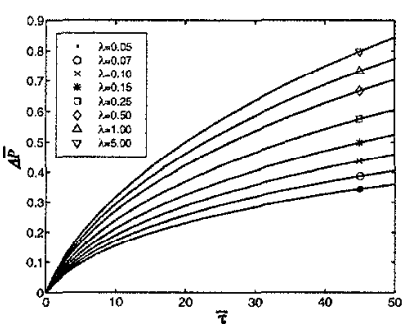

(b)
Fig. 7. Numerically obtained dimensionless (a) power in still fluid and (b) shear-induced power change. the shear-induced power change $\Delta \bar{P}=\bar{P}-\bar{P}_{0}$.

Thus, the heat transfer problem can be solved numerically in terms of these dimensionless parameters, as shown in Fig. 7. Furthermore, this numerical solution can be approximated by the following practically useful closedform expressions. For $0.04 \leq \lambda \leq 5$ and $1 \leq \bar{\tau} \leq 50$, within $5 \%$ error we have

$$
\bar{P}_{0}=1.96 \lambda\left(1+0.435 / \lambda^{0.9}\right)
$$

and

$$
\Delta \bar{P}=\frac{1.31 \lambda^{0.4} \bar{\tau}^{1.47}}{\left(1+2.07 \lambda^{0.6}\right)\left(1+0.56 \bar{\tau}^{0.3} / \lambda^{0.037}\right)^{5}} .
$$

To study the sensors used in our experiments, we can alternatively approximate the numerical solution by

$$
\Delta \bar{P}=\left[0.129 \lambda^{0.45} /\left(1+1.81 \lambda^{0.55}\right)\right] \bar{\tau}^{0.85}
$$

for $0.6 \leq \bar{\tau} \leq 5$, and

$$
\Delta \bar{P}=\left[0.187 \lambda^{0.5} /\left(1+2.1 \lambda^{0.59}\right)\right] \bar{\tau}^{0.67}
$$

for $2.5 \leq \bar{\tau} \leq 25$. In both cases, the applicable range of $\lambda$ is still $0.04 \leq \lambda \leq 5$ and the approximation error is within $10 \%$.

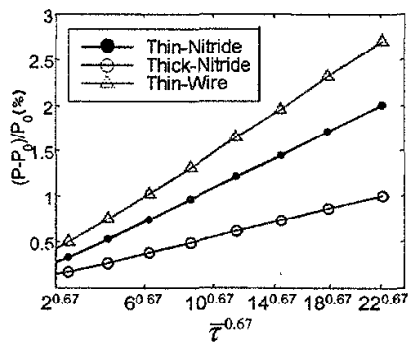

(a)

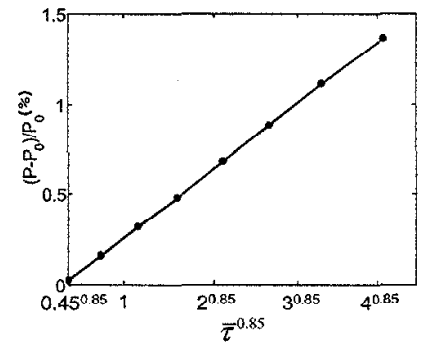

(b)
Fig. 8. Measured shear-induced power vs. (a) $\tau^{0.67}$ for the nitride sensors ( $\tau=1 \mathrm{~Pa}$ gives $\bar{\tau} \approx 22$ ), and (b) $\tau^{0.85}$ for the Parylene sensor ( $\tau=1 \mathrm{~Pa}$ gives $\bar{\tau} \approx 4$ ).

We now plot our experimental data in the appropriate range of $\bar{\tau}$ in Fig. 8. It can be seen that the nitride sensors, and the Parylene sensor, indeed approximately follow the 0.67 th- and 0.85 th-power laws, respectively. This confirms that our 2D theory correctly predicts the trend of MEMS sensor operation.

\section{THREE-DIMENSIONAL MODELING}

Practical MEMS sensors often exhibit significant 3D effects. To obtain quantitative information on such sensors,

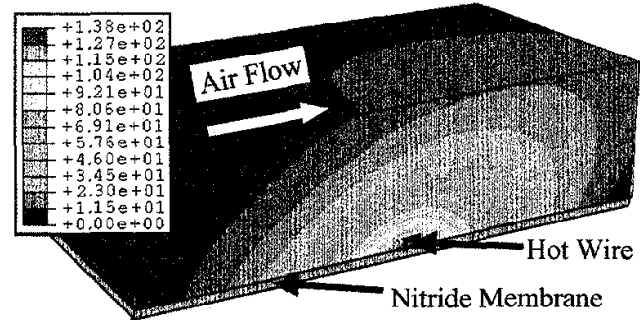

Fig. 9. Temperature distribution in the air flow past the thinnitride sensor operating in constant-tempeature mode with $T_{\mathrm{h}}$ $T_{\infty}=100^{\circ} \mathrm{C} .(\tau=1 \mathrm{~Pa}$ and $P \approx 5.4 \mathrm{~mW}$. 


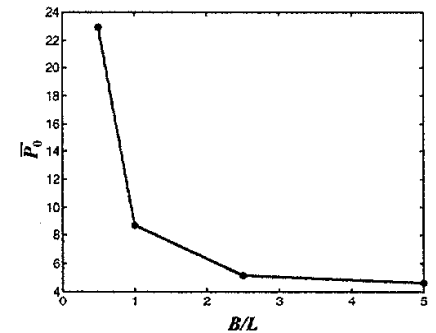

(a)

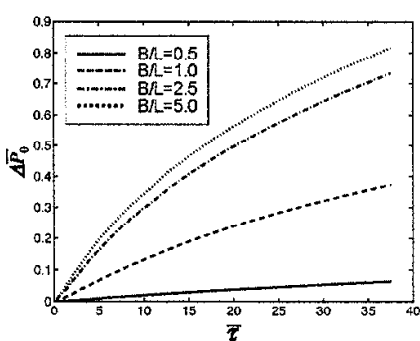

(b)
Fig. 10. (a) Power in still air $\bar{P}_{0}$ and (b) Shear-induced power vs. $\bar{\tau}$ for MEMS sensors for different $B / L$.

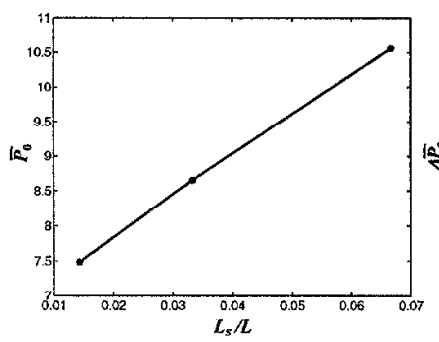

(a)

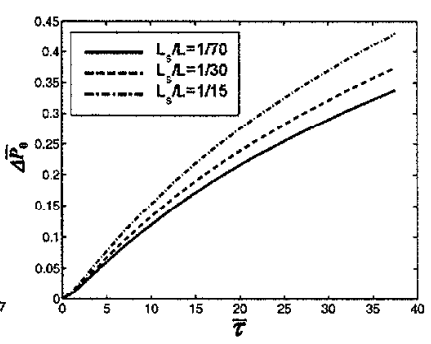

(b)
Fig. 11. (a) Power in still air $\bar{P}_{0}$ and (b) Shear-induced power vs. $\bar{\tau}$ for MEMS sensors for different $L_{s} / L$.

we perform $3 \mathrm{D}$ coupled conduction-convection simulation using the ABAQUS finite element analysis package.

Fig. 9 shows a typical calculated fluid temperature distribution, which clearly indicates that a thin thermal boundary layer does not exist and therefore the classical theory is inappropriate. Figs. 10 and 11 depict the effects of the geometric parameters $B / L$ and $L_{s} / L$ with material properties fixed. Shear stress and sensor power are still nondimensionalized by (6). We see that as the sensor depth $B$ is increased, the dimensionless power consumption in still fluid decreases while the shear-induced power increases. This is consistent with reduced 3D effects for increasing $B$. On the other hand, as the hot wire width increases, both stillfluid and shear-induced powers increase. This is due to the increased contact area between the hot wire and the fluid.

Finally, Fig. 12 compares modeling and experimental

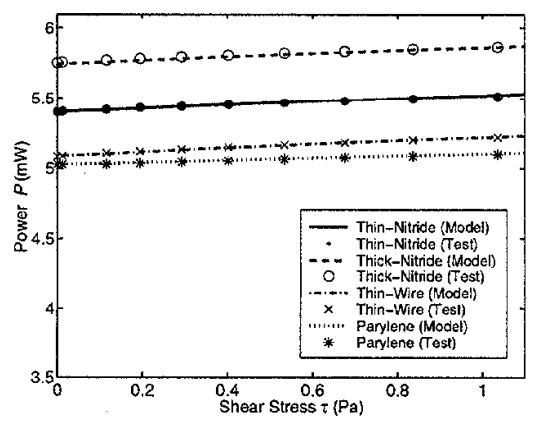

Fig. 12. Comparison of simulation and experimental results (the power for the thick nitride sensor was shifted down by $5.5 \mathrm{~mW}$ for convenience). results for our four MEMS sensors (see the section Experiments). We allow the thermal conductivity of silicon nitride and the height of the Parylene-covered cavity (20 to $40 \mu \mathrm{m})$ to be adjusted in the simulation. It can be seen that the agreement between numerical and testing results is excellent. This confirms that our 3D model provides valid quantitative description of practical MEMS sensors.

\section{CONCLUSION}

We conducted an experimental and theoretical analysis of MEMS thermal shear-stress sensors. The classical theory for macroscale thermal shear-stress sensors was shown to be invalid. A $2 \mathrm{D}$ theory was developed to correctly predict the trend of MEMS sensor operation, and 3D simulation has been performed to provide quantitative description of MEMS sensors. The 2D and 3D models were both compared with experimental data, and yielded excellent qualitative and quantitative agreement.

\section{ACKNOWLEDGMENTS}

The authors appreciate the helpful discussions with Dr. Tim Colonius, Mr. Charles Grosjean, Mr. Wen Hsieh, Mr. Jun Xie, and Mr. Shuyun Wu. This work is supported by AFOSR under Grant F49620-97-1-0514.

\section{REFERENCES}

[1] M. Kimura, S. Tung, J. Lew, C.M. Ho, F. Jiang and Y.C. Tai, "Measurements of Wall Shear Stress of a Turbulent Boundary Layer Using a Micro-Shear-Stress Imaging Chip", Fluid Dynamics Res., Vol. 24, pp. 329-342, 1999.

[2] C. Liu, Y.C. Tai, J.B. Huang and C.M. Ho., "Surface Micromachined Thermal Shear Stress Sensor", 1994 ASME International Mechanical Engineering Congress and Exposition, Chicago, IL, pp. 9-15, 1994.

[3] F. Jiang, Y.C. Tai, B. Gupta, R. Goodman, S. Tung, J.B. Huang and C.M. Ho, "A Micromachined Shear Stress Sensor Imager", MEMS'96, pp. 110-115, 1996.

[4] F. Jiang, Y.C. Tai, K. Walsh, T. Tsao, G.B. Lee and C.M. Ho, "A Flexible MEMS Technology and Its First Application to Shear Stress Sensor Skin," MEMS '97, pp. 465-470, 1997.

[5] X.Q. Wang, Z. Han, F. Jiang, T. Tsao, Q. Lin, Y.C. Tai, V. Koosh, R. Goodman, J. Lew, C.M. Ho, "A Fully Integrated Shear Stress Sensor", Transducers'99, pp. 1074-1077, 1999.

[6] B.J. Bellhouse and D.L. Schultz, "Determination of Mean and Dynamic Skin Friction, Separation and Transition in Low-Speed Flow with a Thin-Film Heated Element", J. Fluid Mechanics, Vol. 24, pp. 379-400, 1966.

[7] F.M. White, Viscous Fluid Flow, $2^{\text {nd }}$ ed., McGraw-Hill, 1991. 